\title{
Construction of the Conserved $\zeta$ via the Effective Action for Perfect Fluids
}

\author{
Junpu Wang \\ Department of Physics and ISCAP, \\ Columbia University, New York, NY 10027, US \\ E-mail: junpu@phys.columbia.edu
}

\begin{abstract}
We consider the problem of how to construct the curvature perturbation $\zeta$, which is expected to be time independent on super-horizon scales to nonlinear level; in particular we concentrate on the case where the existence of such conserved $\zeta$ is guaranteed: a universe dominated by a perfect fluid with adiabatic pressure. We have used a low energy/long wavelength effective action to model the fluid sector and coupled it to the Einstein gravity. This setup enables us to verify explicitly the assumption of "local homogeneity and isotropy" used in previous literature. As a corollary, we also show that the nonlinearly defined graviton field $\gamma_{i j}$ is conserved outside the horizon in the same manner as $\zeta$ is.
\end{abstract}




\section{Contents}

1 Introduction 1

2 Setup 3

2.1 Effective Theory for Perfect Fluids in Flat Spacetime 3

$\begin{array}{lll}2.2 & \text { Cosmological Models with Perfect Fluids } & 7\end{array}$

3 Constructing the Conserved Curvature Perturbation to Non-linear Order

4 Discussion

5 Conclusions

A The Spatially Flat Slicing Gauge (SFSG) to All Orders in Fields $\quad 16$

$\begin{array}{ll}\text { B The Nonlinear Conserved } \zeta \text { in } P(X) \text { Fluids } & 17\end{array}$

\section{Introduction}

Despite the significant success of linear perturbation theory in cosmology over the last few decades, more and more efforts have been devoted in recent years to understanding the cosmological perturbations beyond the linear level - both their origins and evolutions. This is partially motivated by the extraordinary improvement in the accuracy of experiments. For instance, although the result from PLANCK implied the absence of primordial non-Gaussianities, the study on the afterward standard cosmology, such as on the large scale structures, calls for better understanding of the non-linear cosmological perturbations on both the conceptual and the technical/computational level. On the other hand, the observables in cosmology are usually related to gauge invariant quantities, which are rather ambiguous at linear order (since a gauge invariant quantity multiplied by any function of $a(t), H(t)$, etc. is still gauge invariant at linear order). So the study of nonlinear perturbations might give us hints on how observables are related to fundamental physical degrees of freedom.

Among these quantities, enormous attention has been paid to the (linear) curvature perturbation $\zeta$ on uniform Hubble slicings [1]. Equivalently it can thought as the linear scalar metric perturbation on uniform-density hyper-surfaces in [2] and was shown that it is time-independent (conserved) outside the horizon (cf. for instance [3]). Many studies have been done recently seeking to extend the construction of a conserved $\zeta$ to non-linear level in different contexts. Roughly speaking, there are three classes of methods employed to achieve this, which are summarized as follows: 
- The first class is the standard perturbative approach - a perturbative expansion in fields [4]. It is straightforward and the equations governing the evolution of $\zeta$ are valid on all scales. However, the price to pay is that the equations usually become cumbersome once one goes beyond the linear order in the fields and there is a lack of systematic ways to demonstrate the conservation of $\zeta$ on super-horizon scales to arbitrary orders in the field expansion.

- The second class of approaches instead focuses on the spatial gradient expansion that is, an expansion in powers of $\sigma \equiv k / a H$, where $k$ denotes the wavenumber of each Fourier mode. At leading order of this expansion, it corresponds to the separate universe approach. Refs. [5, 6] investigated the cases with an inflating background. There the authors showed that in single field inflation models, $\zeta$ can be defined as the (only) dynamical scalar metric perturbation in the unitary gauge (in which the matter field is demanded to be unperturbed), and that such a $\zeta$ was conserved to all orders in fields on super-horizon scales. The proofs were based on a Lagrangian formalism, for the matter content in these cases was specified by explicit matter actions. On the other hand, the cases with a general FRW background were studied using the formalism developed in [7-9] (known as the $\delta N$ formalism). It was shown that the conservation of the nonlinear $\zeta$ on large scales was assured if the pressure of the matter content was only a function of the energy density or if the matter content could be modeled by a single scalar field. As opposed to the aforementioned field theoretic formalism used in inflation scenarios, these analyses relied on the field equations of motion; in the context of the Einstein gravity the energy conservation alone would be sufficient.

- A third approach was proposed in $[10,11]$ by invoking the purely geometrical description of the curvature perturbation. The covariant quantity constructed there satisfied an exact, non-perturbative, valid-to-all-scale conservation equation and would eventually reduce to the usual $\zeta$ on large scales. The equivalence between this covariant formalism and the $\delta N$ formalism was established in $[12,13]$.

In this note, we will present a systematic way of constructing the conserved curvature perturbation $\zeta$ in a universe dominated by a perfect fluid to all orders in the fields. A low energy effective action for an ordinary perfect fluid $[14,15]$ will be employed to model the matter content. So our method here is parallel to that used in the single field inflation cases $[5,6]$. On the other hand, although this scenario was investigated to some extent in previous literature, we believe that our approach has its own merits in deepening the understanding of this issue from some other aspects: It was crucial for Refs. $[7,8,16]$ to assume that the universe, when it is probed by a sufficiently long wavelength mode, can approximately be thought of as being composed of homogeneous and isotropic patches, each of which looks like an FRW universe. With this very strong assumption, the analysis there can be applied to rather general situations, without even specifying the gravity theory $[7,8,16]$. While in our method, since we specify that the fluid is coupled to the Einstein gravity, we can, in principle, test the assumptions of "local homogeneity and isotropy" using the knowledge of 
the Lagrangian of this dynamical system. Indeed, by applying the Einstein field equations, we can actually show that there exists such a coordinate (or, a gauge choice) that "local homogeneity and isotropy" is manifest. Furthermore our approach enables us to construct the vector and tensor counterparts of $\zeta$ - i.e. the nonlinear vector and tensor perturbations that are time-independent outside the horizon. ${ }^{1}$

Recently, a lot of progress has been made in the study of fluid dynamics via a Lagrangian description $[14,15,17,18]$. Compared to the usual equation-of-motion-based method in this field, one obvious advantage of this approach is its efficiency, - one needs only a single scalar quantity (the Lagrangian) instead of four or more. (For instance, the relativistic fluid with conserved baryon number is governed by the energy-momentum conservation equation and number conservation equation.) In addition, equipped with the knowledge of a field theoretic action, certain fluid dynamical processes can be understood in analogue to scattering experiments, and hence can be dealt with in a straightforward manner [19]. This note applies this method to cosmological fluids. In literature, cosmological fluids are mostly assumed to be irrotational, - which is self consistent when no anisotropy stress is present - , and thus can be modeled as a single scalar field, with the velocity field given by its gradient. However, we will use here a more general Lagrangian description for fluids, which intrinsically includes both gradient and rotational modes. While it is equivalent to the usual scalar description when rotational modes are absent, it enables us also couples to other potential anisotropic sources.

\section{Setup}

We begin with a brief review of the low energy effective description of an ordinary perfect fluid system in Minkowskian spacetime, which was proposed and developed in [14, 15, 17, 18]. For the purpose of this paper, we focus on a fluid with no conserved charges and neglect all the dissipative effects. ${ }^{2}$ The readers already familiar with our notations are welcome to skip directly to Section 2.2, in which the effective fluid action is used as the matter action in the cosmological context and some useful formulas are derived in preparation for the construction of the conserved (nonlinear) quantities on super-horizon scales.

\subsection{Effective Theory for Perfect Fluids in Flat Spacetime}

Our goal is to construct a Lorentz invariant low energy effective theory for an ordinary perfect fluid system. For an ordinary fluid system with no conserved charges, we can specify as the long wavelength degrees of freedom the comoving coordinates of fluid volume elements, parametrized by $\phi^{I}$, with $I=1,2,3$. At any fixed time $t$, the physical position

\footnotetext{
${ }^{1}$ The conservation of the vector field does not contradict the usual intuition that vector modes decay in the absence of anisotropic stress tensor. The time-independent piece of the vector perturbation is of leading order in the spatial gradient expansion. As we will see later, due to the symmetry requirements of a fluid, any constant (in time) transverse vector field configuration is not physical and hence can be set to vanish. The next-to-leading order term of the vector perturbation in the spatial gradient expansion corresponds to the usual decaying configuration.

${ }^{2}$ Roughly speaking, the reason for using a fluid without conserved charges is that, in a universe in thermal equilibrium with all conserved quantum numbers vanishing, the existence of adiabatic mode $\zeta$ is guaranteed [20].
} 
occupied by each volume element is given by $\vec{x}\left(\phi^{I}, t\right)$. In this description, known as the Eulerian description, the physical spatial coordinates $x^{i}$ serve as dynamical fields while $t$ and $\phi^{I}$ are analogous to world sheet coordinates.

However, it is often more convenient to use the inverse functions $\phi^{I}(\vec{x}, t)\left(^{3}\right)$ as dynamical degrees of freedom (this is known as the Lagrangian description), since the spacetime symmetry can be straightforwardly implemented - we simply demand that $\phi^{I}$ transform as scalars under Poincaré transformations. Furthermore we are allowed to choose the comoving coordinates in such a manner that when the fluid system is at rest, in equilibrium and in a homogeneous state at some given external pressure, $\phi^{I}=x^{I}$ - in the field theoretical language, this is equivalent to specifying the ground state of our theory to be

$$
\left\langle\phi^{I}\right\rangle=x^{I}
$$

What are the other symmetries, in addition to the Poincaré invariance, required to make the system behave like an ordinary fluid? Notice that our choice of the ground state (2.1) breaks both spatial translational and rotational invariance. In order for the energy momentum tensor of the system in equilibrium to remain homogeneous and isotropic, as is indeed the case for a fluid, we shall impose internal symmetries to compensate the spontaneously broken spacetime symmetries. More precisely, we demand that the theory be invariant under the internal transformations (with all fields evaluated at the same spacetime point):

$$
\begin{array}{ll}
\mathcal{T}_{i}: \phi^{I} \rightarrow \phi^{\prime I}=\phi^{I}+a^{I}, & a^{I} \text { constant } \\
\mathcal{R}_{i}: \phi^{I} \rightarrow \phi^{I}=O^{I}{ }_{J} \phi^{J}, & O_{J}^{I} \in S O(3)
\end{array}
$$

It is easy to show that the ground state configuration (2.1) is invariant under the diagonal translation and rotation, respectively, which are defined as a linear combination of the spatial and internal transformations : $\mathcal{T}_{d} \equiv \mathcal{T}_{s}+\mathcal{T}_{i}$ and $\mathcal{R}_{d} \equiv \mathcal{R}_{s}+\mathcal{R}_{i} ;{ }^{4}$ it is these residual symmetries that correspond to the homogeneity and isotropy of our background configuration.

Moreover one notices that an ordinary fluid is insensitive to incompressional deformations - it costs no energy to displace fluid volume elements if they are not compressed or dilated. Expressed in terms of a symmetry requirement, we demand that the theory be invariant under volume preserving differomorphisms of the comoving coordinates, defined as

$$
\mathcal{D}: \phi^{I} \rightarrow \xi^{I}(\vec{\phi}), \quad \text { with } \operatorname{det} \frac{\partial \xi^{I}}{\partial \phi^{J}}=1 .
$$

\footnotetext{
${ }^{3}$ For a space filling fluid, there is a diffeomorphism between the comoving coordinates $\phi^{I}$ and the physical spatial coordinates $x^{i}$, so that for any fixed time $t$, the inverse functions $\phi^{I}(\vec{x}, t)$ exist.

${ }^{4}$ The spatial translation $\mathcal{T}_{s}$ and spatial rotation $\mathcal{R}_{s}$ are defined in an analogous fashion as (2.2) (2.3):

$$
\begin{array}{ll}
\mathcal{T}_{s}: x^{I} \rightarrow x^{I I}=x^{I}+a^{I}, & a^{I} \text { constant } \\
\mathcal{R}_{s}: x^{I} \rightarrow x^{I}=O^{I}{ }_{J} x^{J}, & O^{I}{ }_{J} \in S O(3)
\end{array}
$$


So now we are ready to construct the effective action for an ordinary fluid from the $\phi^{I}$, compatible with the symmetry properties mentioned above. It is organized as a derivative expansion. Since the internal translation symmetry (2.2) mandates that each field be accompanied by at least one derivative, at the leading order in the derivative expansion, the only invariant is

$$
B \equiv \operatorname{det}\left(B^{I J}\right), \quad \text { with } B^{I J}=\partial_{\mu} \phi^{I} \partial^{\mu} \phi^{J}
$$

and hence the effective action takes the form of

$$
S_{\text {Fluid }}=\int \mathrm{d}^{4} x F(B)
$$

where $F$ is a generic function, which, as we will see, characterizes the equation of state of the fluid in question.

To illustrate that this effective action indeed describes a perfect fluid, we need to check that the (conserved) energy-momentum tensor takes the famous form:

$$
T_{\mu \nu}=(\rho+p) u_{\mu} u_{\nu}+p \eta_{\mu \nu}
$$

Before doing that let us work out the four-velocity field $u^{\mu}(x)$ for the fluid system in our field theoretical language, which by definition is given by

$$
0=\frac{d}{d \tau} \phi^{I}(x) \equiv u^{\mu}(x) \partial_{\mu} \phi^{I}(x)
$$

where $\tau$ parametrizes the streamline and the derivative with respect to $\tau$ vanishes because the comoving coordinate (or the label) of each fluid volume element is fixed. By solving the above equation for $u^{\mu}$, we obtain

$$
u^{\mu}=-\frac{1}{\sqrt{B}} \epsilon^{\mu \alpha \beta \gamma} \partial_{\alpha} \phi^{1} \partial_{\beta} \phi^{2} \partial_{\gamma} \phi^{3}
$$

where $\epsilon$ is the $4 \mathrm{~d}$ Levi-Civita symbol, with the convention $\epsilon_{0123}=-\epsilon^{0123}=1$. The normalization and overall sign of $u^{\mu}$ are chosen such that $u^{\mu} u_{\mu}=-1$ and $u^{0}>0$.

The energy momentum tensor following from the effective action (2.8) reads

$$
T_{\mu \nu}=-2 F^{\prime}(B) B\left(B^{-1}\right)_{I J} \partial_{\mu} \phi^{I} \partial_{\nu} \phi^{J}+\eta_{\mu \nu} F(B) .
$$

With the aid of (2.11), the energy momentum tensor above indeed can be recast into the perfect fluid form (2.9), if we identify $\rho$ and $p$ with

$$
\rho=-F(B), \quad p=F(B)-2 F^{\prime}(B) B .
$$

This also justifies our previous claim that the generic function $F$ determines the equation of state for fluids. For instance, for an ultra-relativistic fluid with $p=\rho / 3$, one has $F(B) \propto B^{2 / 3}$.

In the rest of this subsection, we will consider small fluctuations about the homogeneous 
equilibrium background configuration (2.1); they are associated with Goldstone excitations:

$$
\phi^{I}=x^{I}+\pi^{I}(x) .
$$

Not all the $\pi^{I}$ fields feature propagating wave solutions. This can be seen by expanding the effective Lagrangian (2.8) to quadratic order:

$$
\mathcal{L}^{(2)}=\frac{1}{2} w_{0}\left(\pi_{L}^{2}-c_{s}^{2}\left(\vec{\partial} \pi_{L}\right)^{2}\right)+\frac{1}{2} w_{0} \dot{\vec{\pi}}_{T}^{2}
$$

where $\pi_{L}$ and $\vec{\pi}_{T}$ are the longitudinal (curl-free) and transverse (divergence-free) components of $\pi^{I}$ :

$$
\pi^{I}=\frac{\partial^{I}}{\sqrt{-\partial^{2}}} \pi_{L}+\pi_{T}^{I}
$$

and where $w_{0}$ is the equilibrium enthalpy density $w_{0}=\bar{\rho}+\bar{p}$. The (squared) speed of sound $c_{s}^{2}$ is given by

$$
c_{s}^{2}=\left.\frac{d p}{d \rho}\right|_{B=\bar{B}}=\left.\frac{2 F^{\prime \prime}(B) B+F^{\prime}(B)}{F^{\prime}(B)}\right|_{B=\bar{B}}
$$

Indeed, we see that only the longitudinal Goldstone field $\pi_{L}$ admits the standard propagating mode with finite speed $c_{s}$ and dispersion relation $\omega=c_{s} k$, while the other Goldstone field $\vec{\pi}_{T}$ has a degenerate dispersion relation $\omega=0$ and thus does not propagate. For this reason, we usually interpret $\pi_{L}$ as the sound wave degree of freedom and $\vec{\pi}_{T}$ as the vortex degree of freedom - vortices do not "propagate" at large distances.

It is worth pointing out that after spontaneous breaking, the spacetime symmetries get mixed with the internal ones, so the three Goldstone fields $\pi^{I}$ transform as a vector field under the diagonal $S O(3) .{ }^{5}$ And after the decomposition $(2.16), \pi_{L}$ can be regarded as a scalar field and $\vec{\pi}_{T}$ as a transverse vector field.

To summarize, we have shown in this subsection that the effective action (2.8) describes a perfect fluid. It is easy to see from (2.13) that the pressure of the fluid is only a function of the energy density, i. e. it satisfies the adiabatic pressure condition. We also want to emphasize that in general this effective description involves three scalar (or equivalently, one scalar and one vector) degrees of freedom. However, it is shown in $[14,23]$ that this effective action can be identified with single scalar action with a shift symmetry - which is known as the low energy description of superfluid, if the vortex mode (vector mode) is neglected:

$$
S_{\text {superfluid }}=\int \mathrm{d}^{4} x P\left((\partial \psi)^{2}\right),
$$

where $P$ here is, analogous to $F$ in our case, some arbitrary function determining the equation of state. The analysis of conservation of curvature perturbation in this context is done in Appendix B.

\footnotetext{
${ }^{5}$ For this reason, in the rest of the paper, we will not distinguish the spatial label " $i, j, \ldots$ " from the internal ones " $I, J, \ldots$ ".
} 


\subsection{Cosmological Models with Perfect Fluids}

In cosmology, the matter content is often modeled by perfect fluids. Fortunately, in our field theoretic language, we can straightforwardly write the action of the cosmological model as

$$
S=S_{\mathrm{EH}}+S_{\text {Fluid }}
$$

where the first term on the r.h.s. is just the usual Einstein - Hilbert action for gravity and the matter action $S_{\text {Fluid }}$ is given by the fluid effective action (2.8), with the flat metric $\eta$ replaced by a dynamical one $g$ and the measure by $\sqrt{-g} \mathrm{~d}^{4} x$. We focus on the case where the ground state configurations for the $\phi^{I}$ are still given by (2.1) and that for the metric is the flat FRW metric: $\bar{g}_{\mu \nu}=\operatorname{diag}\left\{-1, a(t)^{2}, a(t)^{2}, a(t)^{2}\right\}$.

The number of dynamical degrees of freedom in question is counted as follows. In addition to the three Goldstone fields $\pi^{I}$ — which, as argued in last subsection, are eventually regrouped into one longitudinal scalar and one transverse vector under the residual $S O(3)$ group - of the fluid sector, the gravity sector introduces one extra 2-polarized degree of freedom - the spin-2 graviton, a traceless transverse tensor field.

The counting of degrees of freedom is made more explicit by removing the gauge redundancy. Using the $\mathrm{ADM}$ variables, one knows that only the spatial part of the metric is dynamical, and in complete generality it can be decomposed as

$$
g_{i j} \equiv h_{i j}=a(t)^{2} \exp \left(A \delta_{i j}+\partial_{i} \partial_{j} \chi+\partial_{i} C_{j}+\partial_{j} C_{i}+D_{i j}\right)
$$

with $C_{i}, D_{i j}$ satisfying $\partial_{i} C_{i}=0$ and $\partial_{i} D_{i j}=D_{i i}=0$. If the four gauge conditions of coordinate transformations are chosen to set $A=0, \chi=0, C_{i}=0\left({ }^{6}\right)$ - which is known as the spatially flat slicing gauge (SFSG) — we are left with $\pi_{L}, \vec{\pi}_{T}$, and $D_{i j}$ as the dynamical degrees of freedom, which characterizes, respectively, the scalar, vector and tensor cosmological perturbation.

For the interests of this paper, it turns out to be most convenient to work in another gauge, called the unitary gauge $(\mathrm{UG})$, in which all the perturbations are absorbed into the metric, leaving the matter fields unperturbed: $\phi^{I}=x^{I}$. Said differently, the spatial coordinates are chosen to coincide with the comoving coordinates of the fluid. Meanwhile the temporal gauge freedom is used to determine the time slices such that the scalar quantity $B$ (defined in Eqn. (2.7)) remains unperturbed on each time slice: $B(t)=a(t)^{-6}$.

The unitary gauge leads to many conceptual and computational simplifications. First of all, with our choice of the spatial coordinates, the worldlines of fluid volume elements coincide with the threads $x^{i}=$ constant. Indeed, this can be seen by considering the spatial components of the velocity field, which vanish since $u^{I} \propto \epsilon^{I a b c} \partial_{a} \phi^{1} \partial_{b} \phi^{2} \partial_{c} \phi^{3}=0$. Second, our choice of time slices coincides with the uniform density slices, for the energy density of the fluid, given by $\rho=-F(B)$, is a function only of $B$ and it is constant on each time slice. Using the ADM variables, we can parametrize the metric as

$$
d s^{2}=-\mathcal{N}^{2} d t^{2}+h_{i j}\left(d x^{i}+N^{i} d t\right)\left(d x^{j}+N^{j} d t\right)
$$

\footnotetext{
${ }^{6}$ We show in Appendix A that this can always be done through a perturbative construction.
} 
and the inverse metric $g^{\mu \nu}$ as

$$
g^{00}=-\frac{1}{\mathcal{N}^{2}}, \quad g^{0 i}=g^{i 0}=\frac{N^{i}}{\mathcal{N}^{2}}, \quad g^{i j}=h^{i j}-\frac{N^{i} N^{j}}{\mathcal{N}^{2}}
$$

where the spatial metric $h_{i j}$ takes the form of (2.20) and $h^{i j}$ is the inverse spatial metric: $h^{i k} h_{k j}=\delta_{j}^{i}$. One can show easily that in the $\mathrm{UG}, B^{I J}=g^{I J}$ and that

$$
B=\operatorname{det} g^{I J}=\frac{1}{\operatorname{det} h_{I J}}\left(1-\frac{N^{I} N_{I}}{\mathcal{N}^{2}}\right)
$$

where the index of $N^{I}\left(N_{I}\right)$ are lowered (raised) by $h_{I J}\left(h^{I J}\right)$. Therefore the time slicing condition can be expressed as

$$
B(t)=a(t)^{-6} \Longleftrightarrow 3 A+\nabla^{2} \chi=\log \left(1-\frac{N^{I} N_{I}}{\mathcal{N}^{2}}\right) .
$$

Now the counting of number of dynamical d.o.f. in the UG is straightforward: since $\mathcal{N}$ and $N^{I}$ are just auxiliary fields and can be determined in terms of $h_{i j}$ after algebraically solving the constraints $\delta S / \delta \mathcal{N}=0, \delta S / \delta N^{i}=0$, Eqn. (2.24) implies that the two scalar functions $A$ and $\chi$ are not independent. So henceforth we shall always regard $\chi$ as being expressed (perturbatively) in terms of the other perturbations in the metric and select $A$, $C_{i}$ and $D_{i j}$ as the dynamical fields. The number of degrees of freedom is in agreement with that in the SFSG.

We are now in a position to expand the effective action (2.19) up to quadratic order. To do this, we need to solve in the UG the constraint equations $\delta S / \delta N=0, \delta S / \delta N^{i}=0$ and the time slicing condition (2.24) up to linear order in the fields. We find that

$$
\begin{aligned}
S_{T}^{(2)} & =\int d t \int_{\vec{k}} \frac{M_{\mathrm{Pl}}^{2}}{8} a(t)^{3}\left(\dot{D}_{i j}^{2}-\frac{k^{2}}{a^{2}} D_{i j}^{2}\right) \\
S_{V}^{(2)} & =\int d t \int_{\vec{k}} a(t)^{3} \frac{-M_{\mathrm{Pl}}^{2} \dot{H} a(t)^{2}}{1-4 \dot{H} a^{2} / k^{2}} \dot{C}_{i}^{2} \\
S_{S}^{(2)} & =\int d t \int_{\vec{k}} a(t)^{3}\left[\frac{9 M_{\mathrm{Pl}}^{2} \dot{H} a(t)^{2}}{4\left(3 \dot{H} a^{2}-k^{2}\right)}\left(\dot{A}-\frac{\dot{H}}{H} A\right)^{2}-\frac{9 M_{\mathrm{Pl}}^{2} \dot{H}}{4}\left(1+\frac{\ddot{H}}{3 H \dot{H}}\right) A^{2}\right] .
\end{aligned}
$$

where the symbol $\int_{\vec{k}}$ is an abbreviation for $\int \frac{d^{3} k}{(2 \pi)^{3}}$, and where we have used the Friedmann equation

$$
\bar{\rho}=-F(\bar{B})=3 M_{\mathrm{Pl}}^{2} H^{2}, \quad \bar{\rho}+\bar{p}=-2 F^{\prime}(\bar{B}) \bar{B}=-2 M_{\mathrm{Pl}}^{2} \dot{H} .
$$

Notice that in the long wavelength limit $\sigma \equiv k / a H \rightarrow 0$, the actions $(2.25-2.27)$ reduce to

$$
S^{(2)}=S_{T}^{(2)}+S_{V}^{(2)}+S_{S}^{(2)} \simeq \int d t \int_{\vec{k}} M_{\mathrm{Pl}}^{2} a(t)^{3}\left[\frac{1}{8} \dot{D}_{i j}^{2}+\frac{1}{4}\left(k \dot{C}_{i}\right)^{2}+\frac{3}{4} \dot{A}^{2}\right]
$$


A few comments are in order: $i$ ) The speed of a sound mode in curved spacetime is given by

$$
c_{s}^{2}=d p /\left.d \rho\right|_{\bar{B}}=-1-\ddot{H} / 3 H \dot{H}
$$

and that of a transverse mode by $c_{T}^{2}=0$. For the theory to be consistent, we need to impose that $c_{s}$ is sub-luminal and real, and it leads to that

$$
-1<\left.\frac{2 F^{\prime \prime}(B) B}{F^{\prime}(B)}\right|_{B=a^{-6}(t)}<0, \quad \text { or equivalently } 1<-\frac{\ddot{H}}{3 H \dot{H}}<2 .
$$

It is also interesting to point out that our effective fluid model (2.19) is not a good candidate for inflation for two reasons (cf. [21] for a more detailed discussion). First, from (2.28) it can be seen that the slow roll inflation with $H \simeq$ constant and $-\dot{H} / H^{2} \ll 1$ requires the fluid to behave effectively like a cosmological constant, that is

$$
F \sim \Lambda+\epsilon \mathcal{F}
$$

where the first term on the r.h.s. $\Lambda$ is a spacetime-independent constant, while the second term is suppressed by slow roll parameters with respect to the first term and contains the fluid structure function $\mathcal{F}$, which determines the equation of state and satisfies (2.31). From the theoretical point of view, this is not the most interesting model for inflation. Secondly, even though we bear with the previous issue, this effective fluid action should not be employed to study the quantum fluctuations during inflation, because it suffers some quantum mechanical subtleties associated with the vortex degrees of freedom [15]. Therefore to have a consistent inflation model with novelty, we have to relax some symmetry requirements for our system and to make the Lagrangian less constraint, that is, we should use less symmetric objects (e.g. solids) as the matter content [21] to drive inflation.

ii) From the infrared (long wavelength limit) quadratic action (2.29), we see that it is appropriate to treat $A \sim k C_{i} \sim D_{i j}$ as being of the same order in the spatial gradient expansion. Also, despite the deceptive appearance, $\nabla^{2} \chi$ is not necessarily of higher order than $A$ in the spatial gradient expansion; in fact, as we will show soon, they are both of leading order. Thus the scalar, vector and tensor metric perturbations in (2.20) are all of the same order in the spatial gradient expansion, i.e.

$$
A \sim k^{2} \chi \sim k C_{i} \sim D_{i j} \sim \mathcal{O}\left(\sigma^{0}\right)
$$

Following Eqn. (2.29), the linearized equations of motion for scalar mode in the long wavelength limit $\sigma=k / a H \rightarrow 0$ read

$$
\ddot{A}_{\text {lin }}+3 H \dot{A}_{\text {lin }}=0,
$$

and the general solution is given by

$$
A_{\text {lin }}=A_{(1)}+A_{(2)} \int_{t_{c}}^{t} \frac{\mathrm{d} t^{\prime}}{a\left(t^{\prime}\right)^{3}}
$$


where $t_{c}$ is some arbitrary reference time. Furthermore, we can compute the gauge invariant quantities (at linear order): the curvature perturbation $\zeta_{\text {lin }}$ and the comoving curvature perturbation $\mathcal{R}_{\text {lin }}[20]$. In this unitary gauge, they become

$$
\begin{aligned}
\zeta_{\operatorname{lin}} & \equiv \frac{A}{2}-H \frac{\delta \rho}{\dot{\bar{\rho}}}=\frac{A}{2}, \\
\mathcal{R}_{\operatorname{lin}} & \equiv \frac{A}{2}+H \delta u \stackrel{k / a H \rightarrow 0}{\sim} \frac{A}{2}-\frac{H}{\dot{H}} \frac{\dot{A}}{2},
\end{aligned}
$$

where we followed the notation in [20] and defined $\delta u$ via the decomposition

$$
T_{0 i}=\bar{p} N_{i}-(\bar{\rho}+\bar{p})\left(\partial_{i} \delta u+\delta u_{i}^{V}\right) .
$$

Thus it follows immediately from Eqn. (2.35) and Eqn. (2.36) that

$$
\begin{aligned}
\zeta_{\text {lin }} & =\frac{A_{(1)}}{2}+\frac{A_{(2)}}{2} \int_{t_{c}}^{t} \frac{\mathrm{d} t^{\prime}}{a\left(t^{\prime}\right)^{3}}, \\
\mathcal{R}_{\text {lin }} & =\frac{A_{(1)}}{2}+\frac{A_{(2)}}{2} \int_{t_{c}}^{t} \frac{\mathrm{d} t^{\prime}}{a\left(t^{\prime}\right)^{3}}-\frac{A_{(2)} H}{2 a(t)^{3} \dot{H}}, \text { for } \frac{k}{a H} \rightarrow 0 .
\end{aligned}
$$

Notice that the classical solution of $\mathcal{R}_{\text {lin }}$ agrees with results in Ref. [22] and Ref. [24] ${ }^{7}$ : it is the solution of the Mukhanov-Sasaki equation. Indeed, with the aid of Eqn. (2.30), it is to see that

$$
\dot{\mathcal{R}}_{\text {lin }}=-\frac{3 A_{(2)} c_{s}^{2} H^{2}}{2 a^{3} \dot{H}}, \quad \text { for } \frac{k}{a H} \rightarrow 0 .
$$

It is also worth pointing out that, by (2.35) and (2.38), in superhorizon scales, $A_{\text {lin }}$ and $\zeta_{\text {lin }}$ have no growing mode, as long as the universe is expanding. It is not true for $\mathcal{R}_{\text {lin }}$. It was noticed in [25] that, in the so-called non-attractor inflationary phase in which $\varepsilon \equiv-\dot{H} / H^{2} \propto a(t)^{-6}$ and $c_{s}^{2}=$ constant, $\mathcal{R}$ is growing outside horizon. Forturnately, this is not relevant for our purpose: we will focus our attention mainly on $A_{\text {lin }}$, and show in next section that the conservation of it on superhorizon scales actually extends to non-linear order.

\section{Constructing the Conserved Curvature Perturbation to Non-linear Or- der}

As mentioned in the previous section, if the matter content of the universe can be modeled as an ordinary perfect fluid, there is only one dynamical scalar field. In the UG it is parametrized by $A(x)$, the (dynamical) scalar perturbation in the spatial metric (cf. Eqn. (2.20)). In this section, we will show that the evolution of $A$ will remain time-independent as long as the mode is outside the horizon $(\sigma=k / a H \lesssim 1)$. The conservation is preserved to all orders in the field expansion; at linear order $A$ coincides with (twice) the usual (linear)

\footnotetext{
${ }^{7}$ Note that our $\mathcal{R}_{\text {lin }}$ is denoted $\zeta$ there.
} 
curvature perturbation. Therefore we define our non-linear extension of the curvature perturbation $\zeta$ by $\zeta=A / 2$.

The proof of the conservation of $\zeta$ on super-horizon scales will proceed in two main steps as follows: firstly we expand the action (2.19) to leading order in the spatial gradient expansion, viz. to order $\mathcal{O}\left(\sigma^{0}\right)$, while keeping all orders in the fields. We show that the infrared action starts with terms involving at least two time derivatives, each of which acts on a different field. Thus the constant (time-independent) configurations of $A, C_{i}, D_{i j}$ are allowed as solutions of the classical equations of motion. And secondly, we show that these constant configurations are stable under small fluctuations - i.e. they are indeed attractors. As we will see the proofs for the scalar, vector and tensor perturbations are essentially identical.

Step 1: To show the conservation of $A, C_{i}, D_{i j}$, we expand the Lagrangian (2.19) up to the first order in the temporal derivative and to the zeroth order in the spatial gradients, while we are keeping all orders in the fields. The main challenge of doing this is to express the non-dynamical quantities, such as $\mathcal{N}, N^{i}$ and $\chi$, in terms of the dynamical ones, by using the time slicing condition (2.24) and the constraint equations which are given by

$$
\begin{aligned}
0=\frac{\delta S}{\delta \mathcal{N}}= & \frac{M_{\mathrm{Pl}}^{2}}{2}\left[R^{(3)}+\mathcal{N}^{-2}\left(6 H^{2}+2 H \operatorname{Tr} \dot{\mathbf{M}}+\frac{1}{4} \operatorname{Tr}\left(\frac{d}{d t} e^{-\mathbf{M}} \frac{d}{d t} e^{\mathbf{M}}\right)+\frac{1}{4}(\operatorname{Tr} \dot{\mathbf{M}})^{2}\right.\right. \\
& \left.\left.+\hat{\nabla}_{i} N^{j}(\ldots)\right)\right]+F(B)+2 F^{\prime}(B) B \frac{N^{i} N_{i}}{\mathcal{N}^{2}-N^{i} N_{i}} \\
0=\frac{\delta S}{\delta N^{i}}= & \hat{\nabla}_{i}\left[-\frac{2 H}{\mathcal{N}}-\frac{\operatorname{Tr} \dot{\mathbf{M}}}{2 \mathcal{N}}+\frac{\hat{\nabla}_{k} N^{k}}{\mathcal{N}}\right]+\hat{\nabla}_{j}\left[\frac{1}{2 \mathcal{N}}\left(e^{-\mathbf{M}} \frac{d}{d t} e^{\mathbf{M}}\right)_{i}^{j}-\frac{\hat{\nabla}^{j} N_{i}+\hat{\nabla}_{i} N^{j}}{2 \mathcal{N}}\right] \\
& -2 F^{\prime}(B) B \frac{\mathcal{N} N_{i}}{\mathcal{N}^{2}-N_{k} N^{k}}
\end{aligned}
$$

where $R^{(3)}$ is the spatial Ricci scalar constructed from $h_{i j}, \hat{\nabla}$ the covariant derivative compatible with $h_{i j}$, and the matrix $\mathbf{M}_{i j}=\log \left(h_{i j} a^{-2}\right)$. The “..." in the first equation stands for terms that are regular in the limit $\sigma \rightarrow 0$ - the form of these is irrelevant in the analysis. ${ }^{8}$ Inspecting the constraint equation (3.2), one finds that $N^{i}$ starts at least at order $\mathcal{O}(\sigma)$. Thus, as far as the super-horizon modes are concerned, all the terms in

\footnotetext{
${ }^{8}$ One may wonder whether it is legitimate to neglect nonlinear terms (in the EoM) involving spatial derivatives, such as $\left(\partial_{i} \zeta\right)^{2}$, in this long wavelength limit. In general those terms will be cut-off dependent and contribute at the "loop level" as oppose to the tree level, to which we are mainly interested in. For instance, the aforementioned term

$$
\lim _{k \rightarrow 0} \int^{\Lambda} \mathrm{d}^{3} q\left(k_{i}-q_{i}\right) q_{i} \zeta(q) \zeta(k-q) \simeq-\Lambda^{2} \zeta^{2}
$$

behaves like a mass term for $\zeta$ (with $\Lambda$ being the cut-off of the effective action). The cut-off dependence implies that this term can not be physical, and it can be argued that it will be canceled by some counter term. This might not always be the case, and therefore a thorough analysis is needed to understand individual contributions from those type terms. Since we know the effective action of the fluid and its coupling to gravity, in principle, we can compute those "loop" effects, as what the authors did for the curvature perturbation in the context of inflation [26]. This analysis of course lies beyond the scope of this paper, and will be left for the future work.
} 
(3.1) with $N^{i}$ or $\hat{\nabla}$ (as well as the $R^{(3)}$ term) can be neglected, which leads to the crucial fact that $\delta \mathcal{N} \equiv \mathcal{N}-1$ in the super-horizon regime starts with terms involving two time derivatives:

$$
\delta \mathcal{N}=\left[1+\frac{1}{24 H^{2}} \operatorname{Tr}\left(\frac{d}{d t} e^{-\mathbf{M}} \frac{d}{d t} e^{\mathbf{M}}\right)\right]^{1 / 2}-1, \quad \sigma \rightarrow 0
$$

Moreover, we see that for each field, there is at most one time derivative acting on it.

On the other hand, the time slicing condition (2.24), in this long wavelength limit, reduces to

$$
\operatorname{Tr} \mathbf{M}=3 A+\nabla^{2} \chi=\mathcal{O}\left(\sigma^{2}\right),
$$

which verifies our claim that $\nabla^{2} \chi$ is of the same order as $A$ in the spatial gradient expansion at the end of the last section.

It then follows immediately that in the super-horizon regime, the Lagrangian (2.19) becomes

$$
\lim _{k / a H \rightarrow 0} \mathcal{L} \simeq 2 a(t)^{3} \mathcal{N} F(B) \simeq-6 a(t)^{3} M_{\mathrm{Pl}}^{2} H^{2}+\sum_{n \geq 2} \mathcal{L}_{n}\left(\phi_{a}\right)
$$

In the last step we have denoted the dynamical fields - A, $C_{i}, D_{i j}$ - collectively by $\phi_{a}$ and the number of fields contained in $\mathcal{L}_{n}$ by the subscript " $n$ ". Notice that schematically the $\mathcal{L}_{n}$ take the form of

$$
\mathcal{L}_{2} \sim Q_{2}(t) G_{2}^{a b} \dot{\phi_{a}} \dot{\phi_{b}}, \quad \mathcal{L}_{n>2} \sim Q_{n}(t) G_{n}^{a b \ldots k l}(\phi) \dot{\phi}_{a} \dot{\phi}_{b} \ldots \dot{\phi}_{k} \dot{\phi}_{l}
$$

where $Q_{n}(t)$ are functions of time consisting of $a(t), H$ et cetera. Besides the irrelevant field-independent term, this long-wavelength Lagrangian starts at the two time derivative level and hence the e.o.m. following from it reads

$$
f_{1}(\phi, \dot{\phi}) \ddot{\phi}_{a}+f_{2}(\phi, \dot{\phi})_{a}^{b} \dot{\phi}_{b}=0
$$

Therefore it indeed admits $A, C_{i}, D_{i j}=$ constant as solutions to the classical equations of motion. Since this IR Lagrangian (3.6) contains all orders in the fields, the conservation of these fields on super-horizon scales must be preserved nonlinearly.

Step 2: Now we show the solutions $A, C_{i}, D_{i j}=$ constant are actually attractors. We just work on the scalar case, since the analysis for the other two is identical. Writing $A$ as $A=A_{0}+\delta A$, owing to the constancy of $A_{0}$, the quadratic action for the fluctuation $\delta A$ in the long-wavelength limit takes the same form as that for $A$, and it is given by

$$
\delta S_{s}^{(2)}=\int d t \int_{\vec{k}} \frac{3 M_{\mathrm{Pl}}^{2}}{4} a(t)^{3} \dot{\delta} \dot{A}^{2}
$$

from which follows the linearized equation of motion for $\delta A$

$$
\ddot{\delta A}+3 H \dot{\delta} \dot{A}=0
$$


It admits two general solutions - one decaying mode and one constant mode:

$$
\delta A_{1}=\int \frac{d t}{a(t)^{3}}, \quad \delta A_{2}=\text { const. }
$$

This thus confirms that the solution $A=$ constant is an attractor.

As we saw, our approach is more powerful in some aspects than those in previous literature, for it enables us to show that the vector perturbation $C_{i}$ and the tensor perturbation $D_{i j}$ are also conserved on super-horizon scales in the same manner as their scalar counterpart $A$. In the next section, we will show that the nonlinearly defined, conserved quantities $\zeta \equiv A / 2$ and $D_{i j}$ we constructed here agree with those in $[4,8]$, up to a time independent spatial coordinate redefinition.

\section{Discussion}

As was illustrated in the section, we have constructed the curvature perturbation $\zeta$ to nonlinear order through two steps: $i$ ) choose a coordinate system such that the spatial coordinates comove with the fluid and that equal time slice coincides with the uniform density slice; $i i)$ define $\zeta$ to be half the coefficient of the term in $\log \left(a^{-2} g_{i j}\right)$ which is proportional to $\delta_{i j}$ (cf. Eqn. (2.20)), i.e.

$$
\zeta=\frac{A}{2}=\frac{1}{4} \nabla^{-2}\left(\delta_{i j} \nabla^{2}-\partial_{i} \partial_{j}\right) \log \left[a^{-2} g_{i j}\right]
$$

We showed that the $\zeta$ thus defined is time-independent in the super-horizon regime to all orders in the fields.

Our result of the conservation of the non-linear curvature perturbation $\zeta$ on large scales agrees with previous literature $[4,8]$. But in our analysis we did not neglect the presence of the vector and tensor perturbations, nor did we start, a priori, with the assumptions of local homogeneity and isotropy. 9 As we showed explicitly, with those cutoff dependent pieces neglected, the spatial metric in $\mathrm{UG}$ on large scales $(\sigma \rightarrow 0)$ is given by

$$
g_{i j}=a(t)^{2} \exp \left[2 \zeta\left(\delta_{i j}-3 \hat{k}_{i} \hat{k}_{j}\right)+\text { vector }+ \text { tensor }+\mathcal{O}(\sigma)\right] .
$$

At a glimpse, one may think that even though the vector and tensor perturbations are neglected, this metric is anisotropic, which is inconsistent with our intuition about cosmological fluids that no anisotropy in the metric will be generated if the fluid is free of anisotropic stress. However, this puzzle results from our non-conventional choice of coordinates: we can perform a further coordinate transformation (starting from the UG)

$$
t \rightarrow t, \quad x^{I} \rightarrow y^{I}=x^{I}+\xi^{I}(\vec{x})
$$

\footnotetext{
${ }^{9}$ In fact having fixed the UG by $\phi^{I}=x^{I}$ and $B=a(t)^{-6}$, we have depleted all (gauge) freedoms of specifying a coordinate system. Furthermore, as we argued earlier, we should not think that the terms in $g_{i j}$ "superficially" with more derivatives such as $\partial_{i} \partial_{j} \chi, \partial_{i} C_{j}$ as being of higher order than $A \delta_{i j}$ in the spatial gradient expansion. As a consequence of this, once the UG is chosen, we are not entitled to assume any property of $g_{i j}$ on large scales.
} 
such that

$$
\phi^{\prime I}(t, \vec{y})=y^{I}+\xi^{\prime I}(\vec{y}), \quad \text { and } g_{i j}^{\prime}(t, \vec{y})=a(t)^{2} e^{2 \zeta^{\prime}(\vec{y})}\left(e^{\gamma^{\prime}(\vec{y})}\right)_{i j}, \quad \text { with } \partial_{i} \gamma_{i j}^{\prime}=\gamma_{i i}^{\prime}=0
$$

where the function $\xi^{I}(\vec{y})$ is obtained by inverting the 3-diffeomorphism $y^{I}(\vec{x})=x^{I}+\xi^{I}(\vec{x})$. Notice that the time-independence of $\xi^{I}(\vec{x})$ follows directly from the time-independence of $A, C_{i}$, and $D_{i j}$ shown in the last section. Since $\zeta$ in (4.2) and $\zeta^{\prime}$ in (4.4) are related by a time-independent coordinate transformation (4.3), they agree at the linear order in fields

$$
\zeta^{\prime}=\zeta+\mathcal{O}\left(\zeta^{2}, \zeta \gamma, \gamma^{2}\right)
$$

Although discrepancy occurs at nonlinear order, it is not hard to see that $\zeta^{\prime}$ is also timeindependent to all orders in fields in the long wavelength limit - the super-horizian conservation for $\zeta^{\prime}$ follows from that for $\zeta$ and $\gamma$.

Notice that a coordinate transformation (4.3) alters neither the time slices nor the threading of the spatial coordinates, because we

$$
u^{\prime I} \propto \epsilon^{I \alpha \beta \gamma} \partial_{\alpha} \phi^{\prime 1} \partial_{\beta} \phi^{\prime 2} \partial_{\gamma} \phi^{\prime 3}=0, \quad u_{I}^{\prime}=g_{0 I} u^{0}=h_{i j} N^{j} u^{0} \sim \mathcal{O}(\sigma) .
$$

Therefore Eqn. (4.4) and Eqn. (4.6) imply that in the new coordinate system and in the super-horizon regime, both the spatial metric $g_{i j}$ and $T_{i j}^{\prime}$ - which is now reduced to $T_{i j}^{\prime}=p g_{i j}^{\prime}$ (cf. Eqn. (2.9)) - are isotropic, if the tensor perturbation is ignored. Of course the spatial metric $g_{i j}^{\prime}$ is also homogeneous, as long as the limit $\sigma \rightarrow 0$ is concerned in which the spatial dependence of $\zeta^{\prime}$ is negligible.

In summary, although convenient for computational purposes, the UG coordinate is not capable of exhibiting local homogeneity and isotropy in the long wavelength limit. Fortunately there exists a new coordinate system, which is indistinguishable from the UG coordinates when only the physical quantities (or macroscopic quantities) such as $\rho, p, u^{i}$ etc. are considered. (The invariance of $u^{i}$ or $u_{i}$ under the coordinate transformation (4.3) was demonstrated in (4.6), while the invariance of $\rho$ and $p$ follows directly from the fact that, in the UG, $\rho$ and $p$ are only functions of time, which is untouched in coordinate transformation (4.3)). By converting to this new coordinates, the existence of homogeneous and isotropic FRW patches on long wavelength limit is verified (in contrast, many previous papers adopted this as one of the key assumptions.). The $\zeta^{\prime}$ in (4.4) is also time-independent on large scales and it agrees with the definition in $[4,8]$.

Before ending this subsection, we want to point out a subtlety about the vector perturbation (associated with the vortex degree of freedom of fluid). As we showed in the last section, on super-horizon scales, the vector field $C_{i}$ is time-independent. However, this does not correspond to a physical vector mode, which we will now explain. Let us transform to the coordinate system specified by (4.4). Owing to the internal symmetry (2.6) of the fluid system, the $\phi^{I}$ configuration in eqn. (4.4) is in fact physically equivalent to the one free of vortex degrees of freedom — by performing a volume preserving diffeomorphism, we can set to zero the divergence-free component in the $\phi^{I}$. After doing that, neither the 
matter fields nor the spatial metric contain vector modes at leading order in the $\sigma \rightarrow 0$ limit. Said differently, the time-independent $C_{i}$ configuration, as the leading order in the spatial gradient expansion, has no physical significance; the well known decaying vector modes in the universe dominated by a perfect fluid come from next-to-leading order (order $\mathcal{O}(\sigma))$, time-dependent terms in $g_{i j}$ in the UG.

As mentioned at the end of Section 2.1, a perfect fluid free of vortex degrees of freedom (divergence-free vector modes) has an equivalent description [14, 22, 23], in which the low energy effective Lagrangian involves only one scalar field, $\mathcal{L}=P(X) \equiv P\left((\partial \psi)^{2}\right)$. Then we can apply the method in Refs. [5,6] directly to a non-inflating background and define the curvature perturbation $\zeta$ as the (only) dynamical scalar metric perturbation in a gauge specified by ${ }^{10}$ :

$$
\psi(t, \vec{x})=\psi_{0}(t), \quad g_{i j}=a^{2} e^{2 \zeta} \exp \left\{\gamma_{i j}\right\}
$$

Details of the proof of the conservation of $\zeta$ on super-horizon scales in the $P\left((\partial \psi)^{2}\right)$ context are collected in Appendix B. The nonlinear $\zeta$ constructed via this logic is identical to the $\zeta^{\prime}$ field in (4.4), hence it agrees with our definition up to a time-independent reshuffle of the spatial coordinates.

\section{Conclusions}

In this paper we have considered the problem of how to extend the definition of the curvature perturbation $\zeta$ to nonlinear levels; in particular we have concentrated on the situation where the universe is dominated by a perfect fluid. We have used a low energy/long wavelength effective action to model the fluid sector and constructed the nonlinear $\zeta$ as follows: i) fix the gauge so that the spatial comoving coordinates of the fluid coincide with the physical ones and that the constant time slices are the uniform density slices; and ii) define $\zeta=A / 2$ where $A$ is the isotropic scalar part in the spatial metric (2.20). We have shown that this $\zeta$ (as well as its tensor counterpart) is conserved outside the horizon to all orders in the fields.

Although this topic has been investigated to some extent in previous literature, we believe that our approach is novel and has its own merits in some aspects. We have specified the gravity theory to be the Einstein gravity and made use of the Einstein field equations. Thus, instead of assuming it as was done in most previous literature, we are able to show that (at tree level) the universe indeed looks like FRW patches (locally homogeneous and isotropic) on sufficiently large scales. We have also constructed the vector (non-physical) and tensor counterparts of $\zeta$, which are conserved in the same manner as $\zeta$ is. Nevertheless, we have shown our definition of $\zeta$ agrees with that in Malik et al. (2004) [4] and in Lyth et al. (2005) [8] up to a time-independent reshuffle of the spatial coordinates .

\footnotetext{
${ }^{10}$ In Refs. [5, 6] this gauge is referred to as the $\zeta$ gauge or the "unitary" gauge in the context of single field inflation. Despite of the name, it is different from the unitary gauge we meant here, i.e. the one in the context of a cosmological fluid.
} 


\section{Acknowledgments}

We are pleased to thank Lasha Berezhiani, Lam Hui, Jared Kaplan and Alberto Nicolis for illuminating discussions. Especially we would like to thank Bart Horn for his careful reading of the manuscript. The work of J. W. is supported by the DOE under contract DE-FG02-92-ER40699.

\section{Appendix}

\section{A The Spatially Flat Slicing Gauge (SFSG) to All Orders in Fields}

In this section, we show that starting from an arbitrary $g_{\mu \nu}(x)=\bar{g}_{\mu \nu}+\delta g_{\mu \nu}$, we can always achieve the non-linear SFSG, specified by

$$
g_{i j}(x)=a(t)^{2} \exp \gamma_{i j}(x), \quad \text { with } \gamma_{i i}=\partial_{i} \gamma_{i j}=0
$$

via some appropriate gauge transformation $x^{\mu} \rightarrow y^{\mu}=x^{\mu}+\xi^{\mu}(x)$. Here we only concentrate on the case most relevant to our analysis, in which $\bar{g}_{\mu \nu}=\operatorname{diag}\left\{-1, a(t)^{2}, a(t)^{2}, a(t)^{2}\right\}$ and $\delta g$ is treated as a small fluctuation around the FRW background. We prove this by using a perturbative construction.

First let us invert the function $y^{\mu}(x)$ and denote it as

$$
x^{\mu}(y)=y^{\mu}+v^{\mu}(y), \quad \text { with } v^{\mu}(y)=\sum_{n=1}^{\infty} v_{(n)}^{\mu}(y)
$$

where $v_{(n)}^{\mu}$ is assumed to be of order $(\delta g)^{n}$. Under this gauge transformation, the spatial metric transforms as

$$
g_{i j}(x) \rightarrow \tilde{g}_{i j}(y)=\frac{\partial\left(y^{\alpha}+v^{\alpha}\right)}{\partial y^{i}} \frac{\partial\left(y^{\beta}+v^{\beta}\right)}{\partial y^{j}} g_{\alpha \beta}(y+v)
$$

Consider $M_{i j}=\log \left(a^{-2} g_{i j}\right)$. At the first order in $\delta g$, it is straightforward to work out $\tilde{M}_{i j}^{(1)}(y)$, which is given by

$$
\tilde{M}_{i j}^{(1)}=a^{-2} \delta g_{i j}+\partial_{i} v_{(1)}^{j}+\partial_{j} v_{(1)}^{i}+2 H v_{(1)}^{0} \delta_{i j}
$$

Notice that any symmetric tensor function $\delta g_{i j}(x)(i, j=1,2,3)$ can always be put in the form of

$$
\delta g_{i j}=a(t)^{2}\left(\mathcal{A} \delta_{i j}+\partial_{i} \partial_{j} \mathcal{B}+\partial_{i} \mathcal{C}_{j}+\partial_{j} \mathcal{C}_{i}+\mathcal{D}_{i j}\right)
$$


with $\mathcal{C}_{i}$ and $\mathcal{D}_{i j}$ satisfying $\partial_{i} \mathcal{C}_{i}=\mathcal{D}_{i i}=\partial_{i} \mathcal{D}_{i j}=0 .{ }^{11}$ Thus by choosing

$$
v_{(1)}^{0}=-\mathcal{A} / 2 H, \quad v_{(1)}^{S}=-\mathcal{B} / 2, \quad v_{T(1)}^{i}=-\mathcal{C}_{i}
$$

where $v^{S}$ and $v_{T}^{i}$ are the longitudinal (curl-free) and transverse (divergence-free) components of $v_{i}: v_{(n)}^{i}=\partial_{i} v_{(n)}^{S}+v_{T(n)}^{i}$, we find that $\tilde{M}_{i j}^{(1)}=\mathcal{D}_{i j}$ - i.e. that only the transverse traceless part survives.

At the second order the expression for $\tilde{M}_{i j}^{(2)}$ is quite cumbersome, but fortunately we do not need the full expression. Since it takes the form of

$$
\tilde{M}_{i j}^{(2)}=\mathcal{S}_{i j}+\partial_{i} v_{(2)}^{j}+\partial_{j} v_{(2)}^{i}+2 H v_{(2)}^{0} \delta_{i j},
$$

by the same argument as above, we can show that all the fields in $\mathcal{S}_{i j}$ can be set to zero except the TT one. And this process can be repeated to all orders in $\delta g$.

Now let $\gamma_{i j}=\sum_{n=1}^{\infty} \tilde{M}_{i j}^{(n)}$, which apparently is also transverse and traceless. The spatial metric in new coordinate system indeed takes the desired form of Eqn. (A.1).

\section{B The Nonlinear Conserved $\zeta$ in $P(X)$ Fluids}

A perfect fluid free of vortex degrees of freedom has a dual low energy effective description involving only one scalar field $\psi[14,23]$. In the cosmological context, the action is given by

$$
S=S_{E H}+\int \sqrt{-g} P(X) ; \quad X \equiv-\partial_{\mu} \psi \partial_{\nu} \psi g^{\mu \nu} .
$$

The energy momentum tensor can be obtained via varying the matter action with respect to the metric

$$
T_{\mu \nu}=2 P^{\prime}(X) \partial_{\mu} \psi \partial_{\nu} \psi+P(X) g_{\mu \nu}
$$

Noticing that the velocity field $u_{\mu}=\partial_{\mu} \psi X^{-\frac{1}{2}}, T_{\mu \nu}$ can be recast into the standard perfect fluid form (2.9) if we identify

$$
\rho=2 X P^{\prime}-P, \quad p=P .
$$

Assuming that the background configuration of the scalar field is only time dependent $\langle\psi(x)\rangle=\psi_{0}(t)$ and that the background metric takes the usual FRW form, the Einstein

\footnotetext{
${ }^{11}$ To see this, we define $\mathcal{A}$ and $\mathcal{B}$ to be the solutions of

$$
3 \mathcal{A}+\nabla^{2} \mathcal{B}=a^{-2} \delta g_{i i} \quad \text { and } \quad \nabla^{2} \mathcal{A}+\nabla^{2} \nabla^{2} \mathcal{B}=a^{-2} \partial_{i} \partial_{j} \delta g_{i j},
$$
}

then define $\mathcal{C}_{i}$ as the solution of

$$
\partial_{j}\left(\mathcal{A}+\nabla^{2} \mathcal{B}\right)+\nabla^{2} \mathcal{C}_{j}=a^{-2} \partial_{i} \delta g_{i j}
$$

and then use eqn. (A.5) to determine $\mathcal{D}_{i j}$. 
equations and the scalar field equation of motion for unperturbed configurations read

$$
\begin{aligned}
& 3 M_{\mathrm{Pl}}^{2} H^{2}=-P+2 P^{\prime} \dot{\psi}_{0}^{2} \\
& M_{\mathrm{Pl}}^{2} \dot{H}=-P^{\prime} \dot{\psi}_{0}^{2} \\
& \left(P^{\prime}+2 P^{\prime \prime} \dot{\psi}_{0}^{2}\right) \ddot{\psi}_{0}+3 H P^{\prime} \dot{\psi}_{0}=0
\end{aligned}
$$

Now let us fix the gauge. For the sake of defining a nonlinear $\zeta$, the unitary gauge is of most convenience:

$$
\psi(x)=\psi_{0}(t), \quad g_{i j}=a(t)^{2} e^{2 \zeta} \exp \gamma_{i j},
$$

We shall show the $\zeta$ so defined is conserved outside the horizon. We adopt a similar logic as before: first show that $\zeta=$ constant is a solution to the classical equation of motion in the long wavelength limit, and then show that this solution is actually an attractor.

Using the ADM variables, the effective action (B.1) becomes

$$
S=\int \mathcal{N} \sqrt{h}\left(\frac{M_{\mathrm{Pl}}^{2}}{2} R^{(3)}+\frac{M_{\mathrm{Pl}}^{2}}{2 \mathcal{N}^{2}}\left(E_{j}^{i} E_{i}^{j}-E^{2}\right)+P(X)\right)
$$

We can then obtain the constraint equations by varying this action with respect to $\mathcal{N}$ and $N^{i}$ :

$$
\begin{aligned}
& R^{(3)}-\frac{1}{\mathcal{N}^{2}}\left\{-6(H+\dot{\zeta})^{2}+4(H+\dot{\zeta}) \hat{\nabla}_{i} N^{i}-\frac{1}{4} \operatorname{Tr}\left(\frac{d}{d t} e^{-\gamma} \frac{d}{d t} e^{\gamma}\right)+\frac{1}{2} \hat{\nabla}^{i} N_{j} \hat{\nabla}^{j} N_{j}\right. \\
&\left.+\frac{1}{2} \hat{\nabla}_{j} N^{i} \hat{\nabla}^{j} N_{i}-\hat{\nabla}_{i} N^{i} \hat{\nabla}_{j} N^{j}-\frac{1}{2}\left(e^{-\gamma} \frac{d}{d t} e^{\gamma}\right)_{j}^{i}\left(\hat{\nabla}^{j} N_{i}+\hat{\nabla}_{i} N^{j}\right)\right\} \\
&+\frac{2}{M_{\mathrm{Pl}}^{2}} P\left(\frac{\dot{\psi}_{0}^{2}}{\mathcal{N}^{2}}\right)-\frac{4}{M_{\mathrm{Pl}}^{2}} \frac{\dot{\psi}_{0}^{2}}{\mathcal{N}^{2}} P^{\prime}\left(\frac{\dot{\psi}_{0}^{2}}{\mathcal{N}^{2}}\right)=0 \\
& \hat{\nabla}_{i}\left[-\frac{2}{\mathcal{N}}(H+\dot{\zeta})+\frac{1}{\mathcal{N}} \hat{\nabla}_{j} N^{j}\right]+\hat{\nabla}_{j}\left[\frac{1}{2 \mathcal{N}}\left(e^{-\gamma} \frac{d}{d t} e^{\gamma}\right)_{i}^{j}-\frac{1}{2 \mathcal{N}} \hat{\nabla}_{i} N^{j}-\frac{1}{2 \mathcal{N}} \hat{\nabla}^{j} N_{i}\right]=0
\end{aligned}
$$

From equation (B.10), we can see that $\hat{\nabla}_{i} N^{j} \sim \dot{\zeta}$ (or $\dot{\gamma}$ ), i.e. that $\nabla_{i} N^{j}$ is of the zeroth order in the spatial gradient expansion in $\sigma=k / a H$. Thus solving $\delta \mathcal{N} \equiv \mathcal{N}-1$ via equation (B.9) up to order $\mathcal{O}\left(\sigma^{0}\right)$, we have

$$
\delta \mathcal{N}=-M_{\mathrm{Pl}}^{2}\left(P-P^{\prime} \dot{\psi}_{0}^{2}+2 P^{\prime \prime} \dot{\psi}_{0}^{4}\right)^{-1}\left(3 H \dot{\zeta}-H \hat{\nabla}_{i} N^{i}\right)+\mathcal{O}\left(\dot{\zeta}^{2}, \dot{\gamma}^{2}, \dot{\zeta} \dot{\gamma}\right)
$$

Plugging this into the action (B.8) and expanding it to $\mathcal{O}\left(\sigma^{0}\right)$ while keeping all orders in 
fields, we have

$$
\begin{aligned}
S & \rightarrow-2 M_{\mathrm{Pl}}^{2} \int \sqrt{h}\left\{3 H^{2}+\dot{H}+\left(3 H \dot{\zeta}-H \hat{\nabla}_{i} N^{i}\right)+\mathcal{O}\left(\dot{\zeta}^{2}, \dot{\gamma}^{2}, \dot{\zeta} \dot{\gamma}\right)\right\} \\
& =-2 M_{\mathrm{Pl}}^{2} \int \frac{d}{d t}\left(a^{3} e^{3 \zeta} H\right)+\mathcal{O}\left(\dot{\zeta}^{2}, \dot{\gamma}^{2}, \dot{\zeta} \dot{\gamma}\right)
\end{aligned}
$$

where in the first equality we have used the background Einstein equations (B.4) (B.5) and the background scalar field equation (B.6) and in the second equality we have neglected the contribution from $\hat{\nabla}_{i} N^{i}$ since it is a boundary term. Therefore we conclude that the effective action in the long wavelength limit starts with terms involving two time derivatives (acting on different fields) and, by the same argument as before, that $\dot{\zeta}=0, \dot{\gamma}=0$ are solutions to the classical equations of motion.

Now we show that the solutions $\zeta=$ constant and $\gamma=$ constant are attractors. As before we consider the fluctuations around these classical solutions and work out the quadratic action for $\delta \zeta$ and $\delta \gamma$. Owing to the constancy of the classical solutions, the quadratic action for fluctuations takes the same form as that for $\zeta$ and $\gamma$ themselves, which can be obtained by solving the constraint equations (B.9) (B.10) up to the linear order in fields, plugging into (B.8) the linearized solutions $\delta \mathcal{N}_{1}, N_{1}^{i}$ and expanding the effective action to quadratic order. Thus we have

$$
\delta S \simeq M_{\mathrm{Pl}}^{2} \int a^{3}\left[\frac{\varepsilon}{c_{s}^{2}} \dot{\delta \zeta}^{2}-\frac{\varepsilon}{a^{2}}\left(\partial_{i} \delta \zeta\right)^{2}\right]+\frac{1}{8}\left[\dot{\delta}_{i j}^{2}-\frac{1}{a^{2}}\left(\partial_{i} \delta \gamma_{j k}\right)^{2}\right]
$$

where $\varepsilon \equiv-\dot{H} / H^{2}$ and $c_{s}$ is the speed of sound defined as

$$
\begin{aligned}
c_{s}^{2} & \left.\equiv \frac{d p}{d \rho}\right|_{X=\dot{\psi}_{0}^{2}}=\frac{P^{\prime}\left(\dot{\psi}_{0}^{2}\right)}{P^{\prime}\left(\dot{\psi}_{0}^{2}\right)+2 \dot{\psi}_{0}^{2} P^{\prime \prime}\left(\dot{\psi}_{0}^{2}\right)} \\
& =-1-\ddot{H} / 3 H \dot{H}
\end{aligned}
$$

Thus, in the long wavelength limit $\sigma \rightarrow 0$, the linearized equation for $\delta \zeta$ (and for $\delta \gamma$ ) possesses two linearly independent solutions, one being time independent and the other decaying (it is crucial to assume that the combination $\varepsilon / c_{s}^{2}$ does not decay too quickly for the scalar case), which confirms our claim that $\zeta=$ constant and $\gamma=$ constant are attractors.

Before ending this section, let us remark that the nonlinear $\zeta$ constructed in the $P(X)$ context agrees with that in the $F(B)$ context. Notice that in unitary gauge (B.7), $X=$ $\dot{\psi}_{0}(t)^{2}(1+\delta \mathcal{N})^{-2}$. Since on super-horizon scales $\delta \mathcal{N} \rightarrow 0$ due to the constancy of $\zeta$ and $\gamma$, the constant time slices in the unitary gauge coincide with the uniform density slices (for $\rho$ is a function of $X$ only). Similarly, since $N^{i}$ vanishes on large scales for the same reason, we have $u^{i} \propto g^{0 i} \propto N^{i} \rightarrow 0$, i.e. the threading of the spatial coordinates is chosen such that the threads $x^{i}=$ constant coincide with the integral curves of the 4 -velocity $u^{\mu}$ (the comoving worldlines). Therefore $\zeta$ defined in $P(X)$ context is identical to $\zeta^{\prime}$ in (4.4). ${ }^{12}$

\footnotetext{
${ }^{12}$ It seems that the proof that $\zeta$, defined in (B.7), is conversed nonlinearly in superhorizon scales in the
} 


\section{References}

[1] J. M. Bardeen, P. J. Steinhardt and M. S. Turner, "Spontaneous Creation of Almost Scale Free Density Perturbations in an Inflationary Universe," Phys. Rev. D 28, 679 (1983).

[2] J. Bardeen, "Gauge-invariant cosmological perturbations," Phys. Rev. D 22, 1882-1905 (1980).

[3] S. Weinberg, "Adiabatic modes in cosmology," Phys.Rev. D67 (2003) 123504 [astro-ph/0302326]

[4] K. A. Malik, and D. Wands, "Evolution of second-order cosmological perturbations", Class. Quant. Grav. 21:L65-L72, 2004, [arXiv:astro-ph/0307055].

[5] J. Maldacena, "Non-Gaussian features of primordial fluctuations in single field inflationary models," JHEP 0305 (2003) 013 [arXiv:astro-ph/0210603v5]

[6] C. Cheung, A. L. Fitzpatrick, J. Kaplan, and L. Senatore, "On the consistency relation of the 3-point function in single field inflation", JCAP 0802: 021, 2008 [arXiv:0709.0295].

[7] A. Naruko, and M. Sasaki, "Conservation of the nonlinear curvature perturbation in generic single-field inflation", Class. Quant. Grav. 28: 072001, 2011, [arXiv:1101.3180].

[8] D. H. Lyth, K. A. Malik, and M. Sasaki, "A General proof of the conservation of the curvature perturbation", JCAP 0505: 004, 2005, [arXiv:astro-ph/0411220].

[9] G. I. Rigopoulos, E. P. S. Shellard, "The Separate Universe Approach and the Evolution of Nonlinear Superhorizon Cosmological Perturbations", Phys. Rev. D 68:123518,2003 [arXiv:astro-ph/0306620]

[10] D. Langlois, and F. Vernizzi, "Conserved non-linear quantities in cosmology", Phys. Rev. D 72 (2005) 103501, [arXiv: astro-ph/0509078].

[11] D. Langlois, and F. Vernizzi, "Evolution of non-linear cosmological perturbations", Phys. Rev. Lett. 95 (2005) 091303, [arXiv:astro-ph/0503416].

[12] T. Suyama, Y. Watanabe, M. Yamaguchi, "Fully non-linear equivalence of delta N and covariant formalisms", Phys. Rev. D 85 (2012) 083504 [arXiv:1201.3163].

[13] A. Naruko, "A general proof of the equivalence between the N and covariant formalisms", Europhys. Lett. 98 (2012) 69001, [arXiv:1202.1516].

[14] S. Dubovsky, T. Gregoire, A. Nicolis and R. Rattazzi, "Null energy condition and superluminal propagation," JHEP 0603, 025 (2006) [hep-th/0512260].

[15] S. Endlich, A. Nicolis, R. Rattazzi and J. Wang, "The Quantum mechanics of perfect fluids," JHEP 1104, 102 (2011) [arXiv:1011.6396 [hep-th]].

[16] D. Wands, K. A. Malik, D. H. Lyth, and A. R. Liddle, "A new approach to the evolution of cosmological perturbations on large scales", Phys. Rev. D 62 (2000) 043527, [arXiv:astro-ph/0003278].

[17] S. Dubovsky, L. Hui, A. Nicolis and D. T. Son, "Effective Field Theory for Hydrodynamics: Thermodynamics, and the Derivative Expansion", [arXiv:1107.0731 [hep-th]].

[18] S. Endlich, A. Nicolis, R. A. Porto, J. Wang, "Dissipation in the effective field theory for hydrodynamics: First order effects", [arXiv:1211.6461 [hep-th]].

$P(X)$ context fails for the non-attractor inflation scenario [25], while the construction in the $F(B)$ fluid model is still valid. Given the equivalence between the $F(B)$ and $P(X)$ descriptions, it will be interesting to investigate this subtlety. We will leave it to furture work. 
[19] S. Endlich and A. Nicolis, "The incompressible fluid revisited: vortex-sound interactions," arXiv:1303.3289 [hep-th].

[20] S. Weinberg, "Cosmology," Oxford, UK: Oxford Univ. Pr. (2008) 593 p

[21] S. Endlich, A. Nicolis, J. Wang, "Solid Inflation", [arXiv:1210.0569 [hep-th]].

[22] L. Boubekeur, P. Creminelli, J. Norena and F. Vernizzi, "Action approach to cosmological perturbations: the 2nd order metric in matter dominance," JCAP 0808, 028 (2008) [arXiv:0806.1016 [astro-ph]].

[23] S. Sabharwal, J. Wang, "Classical Duality Between irrotational ordinary fluids and superfluids, work in progress.

[24] J. Garriga and V. F. Mukhanov, "Perturbations in k-inflation," Phys. Lett. B 458, 219 (1999) [hep-th/9904176].

[25] M. H. Namjoo, H. Firouzjahi and M. Sasaki, "Violation of non-Gaussianity consistency relation in a single field inflationary model," Europhys. Lett. 101, 39001 (2013) [arXiv:1210.3692 [astro-ph.CO]].

[26] L. Senatore and M. Zaldarriaga, "On Loops in Inflation," JHEP 1012, 008 (2010) [arXiv:0912.2734 [hep-th]]. 